\title{
Some new operators for Fermatean fuzzy matrices
}

\section{Silambarasan}

Department of Mathematics, Sri Manakula Vinayagar Engineering College, Madagadipet, Puducherry-605107, India.; sksimbuking@gmail.com

Academic Editor: Tahir Mehmood

Received: 3 March 2021; Accepted: 17 September 2021; Published: 6 October 2021.

\begin{abstract}
In this paper, we define some new operators $[(A \$ B),(A \# B),(A * B),(A \rightarrow B)]$ of Fermatean fuzzy matrices and investigate their algebraic properties. Further, the necessity and possibility operators of Fermatean fuzzy matrices are proved. Finally, we have identified and proved several of these properties, particularly those involving the operator $A \rightarrow B$ defined as Fermatean fuzzy implication with other operators.
\end{abstract}

Keywords: Fermatean fuzzy matrix; Algebraic sum; Algebraic product; Implication operator.

MSC: 03E72, 08A72, 15B15.

\section{Introduction}

$\mathbf{T}$ he concept of an intuitionistic fuzzy matrix (IFM) was introduced by Khan et al., [1] and simultaneously Im et al., [2] to generalize the concept of Thomason's [3] fuzzy matrix. Since the IFM was proposed, it has received a lot of attention in many fields, such as pattern recognition, medical diagnosis, and so on. But if the sum of the membership degree and the nonmembership degree is greater than 1, the IFM is no longer applicable. In [4] using IFM theory, we developed the Pythagorean fuzzy matrix (PFM) $A=\left[\left\langle\zeta_{a_{i j}} \delta_{a_{i j}}\right\rangle\right]$, where the squared sum of its membership degree $\zeta_{a_{i j}} \in[0,1]$ and nonmembership degree $\delta_{a_{i j}} \in[0,1]$ is less than or equal to 1 .

Since the PFM was brought up, it has been widely applied in different fields, such as investment decision making, service quality of domestic airline, collaborative-based recommender systems, and so on. Although the PFM generalizes the IFM, it cannot describe the following decision information. A panel of experts were invited to give their opinions about the feasibility of an investment plan, and they were divided into two independent groups to make a decision. One group considered the degree of the feasibility of the investment plan as 0.8 , while the other group considered the nonmembership degree as 0.7 . It was clearly seen that $0.8+0.7>1,(0.8)^{2}+(0.7)^{2}>1$, and thus it could not be described by IFM and PFM.

After the IFM and PFM theory, many researchers attempted the important role in this theory, [5-17]. To describe such evaluation information, we have developed Fermatean fuzzy matrix (FFM) $A=\left[\left\langle\zeta_{a_{i j}}, \delta_{a_{i j}}\right\rangle\right]$, where represent the $\zeta_{a_{i j}} \in[0,1]$ membership degree and $\delta_{a_{i j}} \in[0,1]$ the non-membership degree for all $i, j$, respectively, and $0 \leq \zeta_{a_{i j}}^{3}+\delta_{a_{i j}}^{3} \leq 1$ [18]. It was clearly seen that $0.8+0.7>1,(0.8)^{2}+(0.7)^{2}>1,(0.8)^{3}+$ $(0.7)^{3} \leq 1$. Then we defined some Fermatean fuzzy operators $\boxplus_{F}, \bigotimes_{F}, @, \cap, \cup$ and properties are considered. In this paper we have developed some new operators for Fermatean fuzzy matrices and discussed several properties.

\section{Preliminaries}

In this section, some basic concepts related to the fuzzy matrix (FM), intuitionistic fuzzy matrix (IFM) and Pythagorean fuzzy matrix (PFM) have been given.

Definition 1. [3] A fuzzy matrix of $A$ of order $m \times n$ is defined as $A=\left(a_{i j}\right)$, where $a_{i j} \in[0,1]$.

Definition 2. [1] An intuitionistic fuzzy matrix (IFM) is a pair $A=\left[\left\langle\zeta_{a_{i j}}, \delta_{a_{i j}}\right\rangle\right]$ of a non negative real numbers $\zeta_{a_{i j}}, \delta_{a_{i j}} \in[0,1]$ satisfying $0 \leq \zeta_{a_{i j}}+\delta_{a_{i j}} \leq 1$ for all $i, j$. 
Definition 3. [4] A Pythagorean fuzzy matrix (PFM) is a pair $A=\left[\left\langle\zeta_{a_{i j}}, \delta_{a_{i j}}\right\rangle\right]$ of non negative real numbers $\zeta_{a_{i j}}, \delta_{a_{i j}} \in[0,1]$ satisfying the condition $0 \leq \zeta_{a_{i j}}^{2}+\delta_{a_{i j}}^{2} \leq 1$, for all $i, j$. Where $\zeta_{a_{i j}} \in[0,1]$ is called the degree of membership and $\delta_{a_{i j}} \in[0,1]$ is called the degree of non-membership.

Definition 4. [18] A Fermatean fuzzy matrix (FFM) is a pair $A=\left[\left\langle\zeta_{a_{i j}}, \delta_{a_{i j}}\right\rangle\right]$ of non negative real numbers $\zeta_{a_{i j}}, \delta_{a_{i j}} \in[0,1]$ satisfying the condition $0 \leq \zeta_{a_{i j}}^{3}+\delta_{a_{i j}}^{3} \leq 1$, for all $i, j$. Where $\zeta_{a_{i j}} \in[0,1]$ is called the degree of membership and $\delta_{a_{i j}} \in[0,1]$ is called the degree of non-membership.

Definition 5. [18] Let $F_{m \times n}$ denote the family of all $F F M s$ for all $i, j$, and $A, B \in F_{m \times n}$ be given as

$$
A=\left[\left\langle\zeta_{a_{i j}}, \delta_{a_{i j}}\right\rangle\right] \text { and } B=\left[\left\langle\zeta_{b_{i j}}, \delta_{b_{i j}}\right\rangle\right]
$$

then

(i) $A \vee B=\left[\left\langle\max \left\{\zeta_{a_{i j}} \zeta_{b_{i j}}\right\}, \min \left\{\delta_{a_{i j}}, \delta_{b_{i j}}\right\}\right\rangle\right]$,

(iii) $A^{C}=\left[\left\langle\left(\delta_{a_{i j}}\right),\left(\zeta_{a_{i j}}\right)\right\rangle\right]$,

(iv) $A \boxplus_{F} B=\left[\left\langle\sqrt[3]{\zeta_{a_{i j}}^{3}+\zeta_{b_{i j}}^{3}-\zeta_{a_{i j}}^{3} \zeta_{b_{i j}}^{3}}, \delta_{a_{i j}} \delta_{b_{i j}}\right\rangle\right]$,

(v) $A \otimes_{F} B=\left[\left\langle\zeta_{a_{i j}} \zeta_{b_{i j}}, \sqrt[3]{\delta_{a_{i j}}^{3}+\delta_{b_{i j}}^{3}-\delta_{a_{i j}}^{3} \delta_{b_{i j}}^{3}}\right\rangle\right]$,

(vi) $A @ B=\left[\left\langle\sqrt[3]{\frac{\zeta_{a_{i j}}^{3}+\zeta_{b_{i j}}^{3}}{2}}, \sqrt[3]{\frac{\delta_{a_{i j}}^{3}+\delta_{b_{i j}}^{3}}{2}}\right\rangle\right]$.

\section{New operators for Fermatean fuzzy matrices}

In this section, we define the new operators for Fermatean fuzzy matrices and investigates the several algebraic properties.

Definition 6. Let $F_{m \times n}$ denote the family of all FFMs for all $i$, $j$, and let $A, B \in F_{m \times n}$ be given as

(i) $A \$ B=\left[\left\langle\sqrt[3]{\zeta_{a_{i j}} \zeta_{b_{i j}}} \sqrt[3]{\delta_{a_{i j}} \delta_{b_{i j}}}\right\rangle\right]$,

(ii) $A \# B=\left[\left\langle\frac{\sqrt[3]{2} \zeta_{a_{i j}} \zeta_{b_{i j}}}{\sqrt[3]{\zeta_{a_{i j}}^{3}+\zeta_{b_{i j}}^{3}}}, \frac{\sqrt[3]{2} \delta_{a_{i j}} \delta_{b_{i j}}}{\sqrt[3]{\delta_{a_{i j}}^{3}+\delta_{b_{i j}}^{3}}}\right\rangle\right]$.

For which we shall accept that if $\zeta_{a_{i j}}=\zeta_{b_{i j}}=0$ then $\frac{\zeta_{a_{i j}} \zeta_{b_{i j}}}{\zeta_{a_{i j}}+\zeta_{b_{i j}}}=0$ and if $\delta_{a_{i j}}=\delta_{b_{i j}}=0$, then $\frac{\delta_{a_{i j}} \delta_{b_{i j}}}{\delta_{a_{i j}}+\delta_{b_{i j}}}=0$.

(iii) $A * B=\left[\left\langle\sqrt[3]{\frac{\zeta_{a_{i j}}^{3}+\zeta_{b_{i j}}^{3}}{2\left(\zeta_{a_{i j}}^{3}+\zeta_{b_{i j}}^{3}+1\right)}}, \sqrt[3]{\frac{\delta_{a_{i j}}^{3}+\delta_{b_{i j}}^{3}}{2\left(\delta_{a_{i j}}^{3}+\delta_{b_{i j}}^{3}+1\right)}}\right\rangle\right]$,

(iv) $A \rightarrow B=\left[\left\langle\max \left\{\delta_{a_{i j}}, \zeta_{b_{i j}}\right\}, \min \left\{\zeta_{a_{i j}}, \delta_{b_{i j}}\right\}\right\rangle\right]$.

Remark 1. Clearly, for each two FFMs A and B, $[(A @ B),(A \$ B),(A \# B),(A * B),(A \rightarrow B)]$ are as yet an FFM. Some basic representations are appear as follows:

- For (i), $0 \leq\left(\sqrt[3]{\frac{\zeta_{a_{i j}}^{3}+\zeta_{b_{i j}}^{3}}{2}}\right)^{3}+\left(\sqrt[3]{\frac{\delta_{a_{i j}}^{3}+\delta_{b_{i j}}^{3}}{2}}\right)^{3}=\frac{\zeta_{a_{i j}}^{3}+\delta_{a_{i j}}^{3}}{2}+\frac{\zeta_{b_{i j}}^{3}+\delta_{b_{i j}}^{3}}{2} \leq \frac{1}{2}+\frac{1}{2}=1$.

- For (ii), if $\delta_{a_{i j}} \geq \zeta_{b_{i j}}$ and $\zeta_{a_{i j}} \geq \delta_{b_{i j}}$, then $0 \leq \max \left\{\delta_{a_{i j}}^{3}, \gamma_{b_{i j}}^{3}\right\}+\min \left\{\zeta_{a_{i j}}^{3}, \delta_{b_{i j}}^{3}\right\} \leq \delta_{a_{i j}}^{3}+\zeta_{a_{i j}}^{3} \leq 1$.

If $\delta_{a_{i j}} \geq \zeta_{a_{i j}}$ and $\zeta_{a_{i j}} \leq \delta_{b_{i j}}$ then $0 \leq \max \left\{\delta_{a_{i j}}^{3} \zeta_{b_{i j}}^{3}\right\}+\min \left\{\zeta_{a_{i j}}^{3}, \delta_{b_{i j}}^{3}\right\} \leq \delta_{a_{i j}}^{3}+\zeta_{a_{i j}}^{3} \leq 1$.

If $\delta_{a_{i j}} \leq \zeta_{b_{i j}}$ and $\zeta_{a_{i j}} \geq \delta_{b_{i j}}$, then $0 \leq \max \left\{\delta_{a_{i j}}^{3} \zeta_{b_{i j}}^{3}\right\}+\min \left\{\zeta_{a_{i j}}^{3}, \delta_{b_{i j}}^{3}\right\} \leq \delta_{b_{i j}}^{3}+\zeta_{b_{i j}}^{3} \leq 1$.

If $\delta_{a_{i j}} \leq \zeta_{b_{i j}}$ and $\zeta_{a_{i j}} \leq \delta_{b_{i j}}$, then $0 \leq \max \left\{\delta_{a_{i j}}^{3} \zeta_{b_{i j}}^{3}\right\}+\min \left\{\zeta_{a_{i j}}^{3}, \delta_{b_{i j}}^{3}\right\} \leq \delta_{b_{i j}}^{3}+\zeta_{b_{i j}}^{3} \leq 1$. 
- For (iii), $0 \leq\left(\sqrt[3]{\zeta_{a_{i j} \zeta_{b_{i j}}}}\right)^{3}+\left(\sqrt[3]{\delta_{a_{i j} \delta_{b_{i j}}}}\right)^{3}=\zeta_{A}(x) \zeta_{B}(x)+\delta_{A}(x) \delta_{B}(x)=\frac{\zeta_{a_{i j}}^{3}+\delta_{a_{i j}}^{3}}{2}+\frac{\zeta_{b_{i j}}^{3}+\delta_{b_{i j}}^{3}}{2} \leq \frac{1}{2}+$ $\frac{1}{2}=1$.

- For (iv), $0 \leq\left(\frac{\sqrt[3]{2} \zeta_{a_{i j}} \zeta_{b_{i j}}}{\sqrt[3]{\zeta_{a_{i j}}^{3}+\zeta_{b_{i j}}^{3}}}\right)^{3}+\left(\frac{\sqrt[3]{2} \delta_{a_{i j}} \delta_{b_{i j}}}{\sqrt[3]{\delta_{a_{i j}}^{3}+\delta_{b_{i j}}^{3}}}\right)^{3}=\frac{2 \zeta_{a_{i j}}^{3} \zeta_{b_{i j}}^{3}}{\zeta_{a_{i j}}^{3}+\zeta_{b_{i j}}^{3}}+\frac{2 \delta_{a_{i j}}^{3} \delta_{b_{i j}}^{3}}{\delta_{a_{i j}}^{3}+\delta_{b_{i j}}^{3}} \leq 1$.

- For (v), $0 \leq\left(\sqrt[3]{\frac{\zeta_{a_{i j}}^{3}+\zeta_{b_{i j}}^{3}}{2\left(\zeta_{a_{i j}}^{3}+\zeta_{b_{i j}}^{3}+1\right)}}\right)^{3}+\left(\sqrt[3]{\frac{\delta_{a_{i j}}^{3}+\delta_{b_{i j}}^{3}}{2\left(\delta_{a_{i j}}^{3}+\delta_{b_{i j}}^{3}+1\right)}}\right)^{3}=\frac{\zeta_{a_{i j}}^{3}+\zeta_{b_{i j}}^{3}}{2\left(\zeta_{a_{i j}}^{3}+\zeta_{b_{i j}}^{3}+1\right)}, \frac{\delta_{a_{i j}}^{3}+\delta_{b_{i j}}^{3}}{2\left(\delta_{a_{i j}}^{3}+\delta_{b_{i j}}^{3}+1\right)} \leq 1$.

Lemma 1. [6] For any two numbers $a, b \in[0,1]$, then

$$
a \cdot b \leq \min \{a, b\} \leq \frac{2(a \cdot b)}{a+b} \leq \sqrt{a \cdot b} \leq \max \{a, b\} \leq a+b-a \cdot b,
$$

and

$$
a . b \leq \frac{a+b}{2(a+b+1)} \leq \frac{a+b}{2} .
$$

Theorem 1. For $A, B \in F_{m \times n}$, we have

(i) $A @ B=B @ A=\left(A^{C} @ B^{C}\right)^{C}$,

(ii) $A \$ B=B \$ A=\left(A^{C} \$ B^{C}\right)^{C}$,

(iii) $A \# B=B \# A=\left(A^{C} \# B^{C}\right)^{C}$,

(iv) $A * B=B * A=\left(A^{C} * B^{C}\right)^{C}$.

Proof. Here, we prove only (i). Others can be proved similarly.

Let $\mathrm{A}$ and $\mathrm{B}$ be two given FFMs, then

$$
\begin{aligned}
& A @ B=\left[\left\langle\sqrt[3]{\frac{\zeta_{a_{i j}}^{3}+\zeta_{b_{i j}}^{3}}{2}}, \sqrt[3]{\frac{\delta_{a_{i j}}^{3}+\delta_{b_{i j}}^{3}}{2}}\right\rangle\right]=\left[\left\langle\sqrt[3]{\frac{\zeta_{b_{i j}}^{3}+\zeta_{a_{i j}}^{3}}{2}}, \sqrt[3]{\frac{\delta_{b_{i j}}^{3}+\delta_{a_{i j}}^{3}}{2}}\right\rangle\right]=B @ A, \\
& A^{C} @ B^{C}=\left[\left\langle\sqrt[3]{\frac{\delta_{a_{i j}}^{3}+\delta_{b_{i j}}^{3}}{2}}, \sqrt[3]{\frac{\zeta_{a_{i j}}^{3}+\zeta_{b_{i j}}^{3}}{2}}\right\rangle\right] \\
& \left(A^{C} @ B^{C}\right)^{C}=\left[\left\langle\sqrt[3]{\frac{\zeta_{a_{i j}}^{3}+\zeta_{b_{i j}}^{3}}{2}}, \sqrt[3]{\frac{\delta_{a_{i j}}^{3}+\delta_{b_{i j}}^{3}}{2}}\right\rangle\right]=A @ B .
\end{aligned}
$$

Hence, $A @ B=B @ A=\left(A^{C} @ B^{C}\right)^{C}$.

The following theorems are obvious:

Theorem 2. For $A, B, C \in F_{m \times n}$, we have
(i) $(A \wedge B) @ C=(A @ C) \wedge(B @ C)$,
(ii) $(A \vee B) @ C=(A @ C) \vee(B @ C)$,
(iii) $(A \wedge B) \$ C=(A \$ C) \wedge(B \$ C)$,
(iv) $(A \vee B) \$ C=(A \$ C) \vee(B \$ C)$,
(v) $(A \wedge B) \# C=(A \# C) \wedge(B \# C)$,
(vi) $(A \vee B) \# C=(A \# C) \vee(B \# C)$,
(vii) $(A \wedge B) * C=(A * C) \wedge(B * C)$,
(viii) $(A \vee B) * C=(A * C) \vee(B * C)$.

Theorem 3. For $A, B, C \in F_{m \times n}$, we have

(i) $\left(A \boxplus_{F} B\right) @ C \leq(A @ C) \boxplus_{F}(B @ C)$,

(ii) $\left(A \rrbracket_{F} B\right) @ C \geq(A @ C) \rrbracket_{F}(B @ C)$, 
(iii) $\left(A \boxplus_{F} B\right) \$ C \leq(A \$ C) \boxplus_{F}(B \$ C)$,

(vi) $\left(A \bigotimes_{F} B\right) \$ C \geq(A \$ C) \bigotimes_{F}(B \$ C)$,

(v) $\left(A \boxplus_{F} B\right) * C \leq(A * C) \boxplus_{F}(B * C)$,

(vi) $\left(A \bigotimes_{F} B\right) * C \geq(A * C) \bigotimes_{F}(B * C)$.

Theorem 4. For $A, B, C \in F_{m \times n}$, we have

(i) $(A @ B) \boxplus_{F} C=\left(A \boxplus_{F} C\right) @\left(B \boxplus_{F} C\right)$,

(ii) $(A @ B) \bigotimes_{F} C=\left(A \bigotimes_{F} C\right) @\left(B \rrbracket_{F} C\right)$,

(iii) $(A \$ B) \boxplus_{F} C \leq\left(A \boxplus_{F} C\right) \$\left(B \boxplus_{F} C\right)$,

(iv) $(A \$ B) \bigotimes_{F} C \geq\left(A \bigotimes_{F} C\right) \$\left(B \nabla_{F} C\right)$,

(v) $(A \# B) \boxplus_{F} C \leq\left(A \boxplus_{F} C\right) \#\left(B \boxplus_{F} C\right)$,

(vi) $(A \# B) \bigotimes_{F} C \geq\left(A \nabla_{F} C\right) \#\left(B \nabla_{F} C\right)$,

(vii) $(A * B) \boxplus_{F} C \leq\left(A \boxplus_{F} C\right) *\left(B \boxplus_{F} C\right)$,

(viii) $(A * B) \bigotimes_{F} C \geq\left(A \rrbracket_{F} C\right) *\left(B \rrbracket_{F} C\right)$.

\section{Necessity and possibility operators on Fermatean fuzzy matrices}

In this section, we prove the necessity and possibility operators of Fermatean fuzzy matrices. Then we compile some relevent properties of these operators are discussed.

Definition 7. [18] The necessity and possibility operators on a Fermatean fuzzy matrix $\mathrm{A}$ is denoted by $\square A, \diamond A$ and is $\square A=\left[\left\langle\zeta_{a_{i j}}, \sqrt[3]{1-\zeta_{a_{i j}}^{3}}\right\rangle\right], \diamond A=\left[\left\langle\sqrt[3]{1-\delta_{a_{i j}}^{3}}, \delta_{a_{i j}}\right\rangle\right]$.

Theorem 5. For $A, B \in F_{m \times n}$, we have

(i) $\square A @ \square B=\square(A @ B) \leq \diamond A @ \diamond B=\diamond(A @ B)$,

(ii) $\square(A \$ B) \leq \square A \$ \square B \leq \diamond A \$ \diamond B \leq \diamond(A \$ B)$,

(iii) $\square(A \# B) \leq \square A \# \square B \leq \diamond A \# \diamond B \leq \diamond(A \# B)$,

(iv) $\square(A * B) \leq \square A * \square B \leq \diamond A * \diamond B \leq \diamond(A * B)$.

Proof. Here we prove (i) and (iii). (ii) and (iv) can be proved similarly.

(i) Since

$$
\square A @ \square B=\left[\left\langle\sqrt[3]{\frac{\zeta_{a_{i j}}^{3}+\zeta_{b_{i j}}^{3}}{2}}, \sqrt[3]{\frac{1-\zeta_{a_{i j}}^{3}+1-\zeta_{b_{i j}}^{3}}{2}}\right\rangle\right]=\square(A @ B),
$$

and

$$
\diamond A @ \diamond B=\left[\left\langle\sqrt[3]{\frac{1-\delta_{a_{i j}}^{3}+1-\delta_{b_{i j}}^{3}}{2}}, \sqrt[3]{\frac{\delta_{a_{i j}}^{3}+\delta_{b_{i j}}^{3}}{2}}\right\rangle\right]=\diamond(A @ B) .
$$

So,

$$
\square A @ \square B=\square(A @ B) \leq \diamond A @ \diamond B=\diamond(A @ B) .
$$

(iii) Since

$$
\square A \# \square B=\left[\left\langle\frac{\sqrt[3]{2} \zeta_{a_{i j}} \zeta_{b_{i j}}}{\sqrt[3]{\zeta_{a_{i j}}^{3}+\zeta_{b_{i j}}^{3}}}, \frac{\sqrt[3]{2} \sqrt[3]{1-\zeta_{a_{i j}}^{3}} \sqrt[3]{1-\zeta_{b_{i j}}^{3}}}{\sqrt[3]{1-\zeta_{a_{i j}}^{3}+1-\zeta_{b_{i j}}^{3}}}\right\rangle\right] \leq \square(A \# B),
$$

and

$$
\diamond A \# \diamond B=\left[\left\langle\frac{\sqrt[3]{2} \sqrt[3]{1-\delta_{a_{i j}}^{3}} \sqrt[3]{1-\delta_{b_{i j}}^{3}}}{\sqrt[3]{1-\delta_{a_{i j}}^{3}+1-\delta_{b_{i j}}^{3}}}, \frac{\sqrt[3]{2} \delta_{a_{i j}} \delta_{b_{i j}}}{\sqrt[3]{\delta_{a_{i j}}^{3}+\delta_{b_{i j}}^{3}}}\right\rangle\right] \leq \diamond(A \# B) .
$$

So,

$$
\square(A \# B) \leq \square A \# \square B \leq \diamond A \# \diamond B \leq \diamond(A \# B) .
$$

The following theorems are obvious: 
Theorem 6. For $A, B \in F_{m \times n}$, we have
(i) $\square\left[(\diamond A @ \diamond B)^{C}\right]=[\diamond(A @ B)]^{C}$,
(ii) $\diamond\left[(\square A @ \square B)^{C}\right]=[\square(A @ B)]^{C}$,
(iii) $\square\left[(\diamond A \$ \diamond B)^{C}\right]=[\diamond(A \$ B)]^{C}$,
(vi) $\diamond\left[(\square A \$ \square B)^{C}\right]=[\square(A \$ B)]^{C}$,
(v) $\square\left[(\diamond A \# \diamond B)^{C}\right]=[\diamond(A \# B)]^{C}$,
(vi) $\diamond\left[(\square A \# \square B)^{C}\right]=[\square(A \# B)]^{C}$,
(vii) $\square\left[(\diamond A * \diamond B)^{C}\right]=[\diamond(A * B)]^{C}$,
(viii) $\diamond\left[(\square A * \square B)^{C}\right]=[\square(A * B)]^{C}$.

Theorem 7. For $A, B \in F_{m \times n}$, we have
(i) $\left[\left(\square A \boxplus_{F} \diamond B\right)^{C} @\left((\square A)^{C} \otimes_{F} \diamond B\right)\right] \vee(\square A)^{C}=(\square A)^{C}$,
(ii) $\left[\left(\square A \nabla_{F} \diamond B\right)^{C} @\left((\square A)^{C} \boxplus_{F} \diamond B\right)\right] \wedge(\square A)^{C}=(\square A)^{C}$,
(iii) $\left.\left(\square A \boxplus_{F} \diamond B\right)^{C} \$\left((\square A)^{C} \otimes_{F} \diamond B\right)\right] \vee(\square A)^{C}=(\square A)^{C}$,
(iv) $\left[\left(\square A \otimes_{F} \diamond B\right)^{C} \$\left((\square A)^{C} \boxplus_{F} \diamond B\right)\right] \wedge(\square A)^{C}=(\square A)^{C}$,
(v) $\left[\left(\square A \boxplus_{F} \diamond B\right)^{C} \#\left((\square A)^{C} \bigotimes_{F} \diamond B\right)\right] \vee(\square A)^{C}=(\square A)^{C}$,
(vi) $\left[\left(\square A \bigotimes_{F} \diamond B\right)^{C} \#\left((\square A)^{C} \boxplus_{F} \diamond B\right)\right] \wedge(\square A)^{C}=(\square A)^{C}$,
(vii) $\left[\left(\diamond A \boxplus_{F} \square B\right)^{C} @\left((\square A)^{C} \nabla_{F} \diamond B\right)\right] \vee(\diamond A)^{C}=(\diamond A)^{C}$,
(viii) $\left[\left(\diamond A \boxplus_{F} \square B\right)^{C} \$\left((\square A)^{C} \otimes_{F} \diamond B\right)\right] \vee(\diamond A)^{C}=(\diamond A)^{C}$,
(ix) $\left[\left(\diamond A \boxplus_{F} \square B\right)^{C} \#\left((\square A)^{C} \otimes_{F} \diamond B\right)\right] \vee(\diamond A)^{C}=(\diamond A)^{C}$,
(x) $\left[\left(\diamond A \boxplus_{F} \square B\right)^{C} @\left((\diamond A)^{C} \bigotimes_{F} \square B\right)\right] \vee(\diamond A)^{C}=(\diamond A)$,
(xi) $\left[\left(\diamond A \boxplus_{F} \square B\right)^{C} \$\left((\diamond A)^{C} \nabla_{F} \square B\right)\right] \vee(\diamond A)^{C}=(\diamond A)$,
(xii) $\left[\left(\diamond A \boxplus_{F} \square B\right)^{C} \#\left((\diamond A)^{C} \otimes_{F} \square B\right)\right] \vee(\diamond A)^{C}=(\diamond A)$.

In the next section, we state and prove some new results involving implication operator with other FFM operators

\section{Some results of FFMs based on implication operator}

In this section, the proofs of the following theorems and corollaries follows from the Definitions 5, 6 and Lemma 1.

Theorem 8. For $A, B \in F_{m \times n}$, we have
(i) $\left(A^{C} \rightarrow B\right) @\left(A \rightarrow B^{C}\right)^{C}=(A @ B)$,
(ii) $\left(A^{C} \rightarrow B\right) \boxplus_{F}\left(A \rightarrow B^{C}\right)^{C}=\left(A \boxplus_{F} B\right)$,
(iii) $\left(A^{C} \rightarrow B\right) \bigotimes_{F}\left(A \rightarrow B^{C}\right)^{C}=\left(A \bigotimes_{F} B\right)$,
(iv) $\left(A^{C} \rightarrow B\right) \$\left(A \rightarrow B^{C}\right)^{C}=(A \$ B)$,
(v) $\left(A^{C} \rightarrow B\right) \#\left(A \rightarrow B^{C}\right)^{C}=(A \# B)$,
(vi) $(A \rightarrow B)^{C} \boxplus_{F}(B \rightarrow A)=\left(A \boxplus_{F} B^{C}\right)$,
(vii) $(A \rightarrow B)^{C} @(B \rightarrow A)=\left(A @ B^{C}\right)$,
(viii) $(A \rightarrow B)^{C} \bigotimes_{F}(B \rightarrow A)=\left(A \bigotimes_{F} B^{C}\right)$,
(ix) $(A \rightarrow B)^{C} \$(B \rightarrow A)=\left(A \$ B^{C}\right)$,
(x) $(A \rightarrow B)^{C} \#(B \rightarrow A)=\left(A \# B^{C}\right)$.

Proof. We prove only (i) and (vi) and remaining can be proved analogously. 
(i)

$$
\begin{aligned}
\left(A^{C} \rightarrow B\right) @ & \left(A \rightarrow B^{C}\right)^{C} \\
& =\left[\sqrt[3]{\frac{\max \left\{\zeta_{a_{i j}}^{3} \zeta_{b_{i j}}^{3}\right\}+\min \left\{\zeta_{a_{i j}}^{3} \zeta_{b_{i j}}^{3}\right.}{2}}, \sqrt[3]{\left.\frac{\min \left\{\delta_{a_{i j}}^{3}, \delta_{b_{i j}}^{3}\right\}+\max \left\{\delta_{a_{i j}}^{3} \delta_{b_{i j}}^{3}\right\}}{2}\right]}\right. \\
& =\left[\left\langle\sqrt[3]{\frac{\zeta_{a_{i j}}^{3}+\zeta_{b_{i j}}^{3}}{2}}, \sqrt[3]{\left.\left.\frac{\delta_{a_{i j}}^{3}+\delta_{b_{i j}}^{3}}{2}\right\rangle\right]}\right.\right. \\
& =(A @ B) .
\end{aligned}
$$

(vi)

$$
\begin{aligned}
& (A \rightarrow B)^{C} \boxplus_{F}(B \rightarrow A) \\
& =\left[\sqrt[3]{\min \left\{\zeta_{a_{i j}}^{3}, \delta_{b_{i j}}^{3}\right\}+\max \left\{\delta_{b_{i j}}^{3} \zeta_{a_{i j}}^{3}\right\}-\min \left\{\zeta_{a_{i j}}^{3}, \delta_{b_{i j}}^{3}\right\} \max \left\{\delta_{b_{i j}}^{3}, \zeta_{a_{i j}}^{3}\right\}}, \max \left\{\delta_{a_{i j}} \zeta_{b_{i j}}\right\} \min \left\{\zeta_{b_{i j}}, \delta_{a_{i j}}\right\}\right] \\
& =\left[\left\langle\sqrt[3]{\zeta_{a_{i j}}^{3}+\delta_{b_{i j}}^{3}-\zeta_{a_{i j}}^{3} \delta_{b_{i j}}^{3}} \delta_{a_{i j}} \zeta_{b_{i j}}\right\rangle\right] \\
& =\left(A \boxplus_{F} B^{C}\right) .
\end{aligned}
$$

Theorem 9. For $A, B \in F_{m \times n}$, we have
(i) $\left(\left(A \boxplus_{F} B\right) \rightarrow(A @ B)^{C}\right)^{C}=\left((A @ B) \rightarrow\left(A \boxplus_{F} B\right)^{C}\right)^{C}=(A @ B)$,
(ii) $\left(\left(A \boxplus_{F} B\right)^{C} \rightarrow(A @ B)\right)=\left((A @ B)^{C} \rightarrow\left(A \boxplus_{F} B\right)\right)=\left(A \boxplus_{F} B\right)$,
(iii) $\left(\left(A \bigotimes_{F} B\right) \rightarrow(A @ B)^{C}\right)^{C}=\left((A @ B) \rightarrow\left(A \bigotimes_{F} B\right)^{C}\right)^{C}=\left(A \bigotimes_{F} B\right)$,
(iv) $\left(\left(A \rrbracket_{F} B\right)^{C} \rightarrow(A @ B)\right)=\left((A @ B)^{C} \rightarrow\left(A \bigotimes_{F} B\right)\right)=(A @ B)$,
(v) $\left(\left(A \boxplus_{F} B\right) \rightarrow(A \# B)^{C}\right)^{C}=\left((A \# B) \rightarrow\left(A \boxplus_{F} B\right)^{C}\right)^{C}=(A \# B)$,
(vi) $\left(\left(A \boxplus_{F} B\right)^{C} \rightarrow(A \# B)\right)=\left((A \# B)^{C} \rightarrow\left(A \boxplus_{F} B\right)\right)=\left(A \boxplus_{F} B\right)$,
(vii) $\left(\left(A \bigotimes_{F} B\right) \rightarrow(A \# B)^{C}\right)^{C}=\left((A \# B) \rightarrow\left(A \nabla_{F} B\right)^{C}\right)^{C}=\left(A \nabla_{F} B\right)$,
(viii) $\left(\left(A \nabla_{F} B\right)^{C} \rightarrow(A \# B)\right)=\left((A \# B)^{C} \rightarrow\left(A \bigotimes_{F} B\right)\right)=(A \# B)$,
(ix) $\left(\left(A \boxplus_{F} B\right) \rightarrow(A \$ B)^{C}\right)^{C}=\left((A \$ B) \rightarrow\left(A \boxplus_{F} B\right)^{C}\right)^{C}=(A \$ B)$,
(x) $\left(\left(A \boxplus_{F} B\right)^{C} \rightarrow(A \$ B)\right)=\left((A \$ B)^{C} \rightarrow\left(A \boxplus_{F} B\right)\right)=\left(A \boxplus_{F} B\right)$,
(xi) $\left(\left(A \bigotimes_{F} B\right) \rightarrow(A \$ B)^{C}\right)^{C}=\left((A \$ B) \rightarrow\left(A \nabla_{F} B\right)^{C}\right)^{C}=\left(A \bigotimes_{F} B\right)$,
(xii) $\left(\left(A \nabla_{F} B\right)^{C} \rightarrow(A \$ B)\right)=\left((A \$ B)^{C} \rightarrow\left(A \nabla_{F} B\right)\right)=(A \$ B)$,
(xiii) $\left(\left(A \boxplus_{F} B\right) \rightarrow\left(A \rrbracket_{F} B\right)^{C}\right)^{C}=\left(\left(A \rrbracket_{F} B\right) \rightarrow\left(A \boxplus_{F} B\right)^{C}\right)^{C}=\left(A \rrbracket_{F} B\right)$,
(xiv) $\left(\left(A \rrbracket_{F} B\right)^{C} \rightarrow\left(A \boxplus_{F} B\right)\right)=\left(\left(A \boxplus_{F} B\right)^{C} \rightarrow\left(A \boxplus_{F} B\right)\right)=\left(A \boxplus_{F} B\right)$.

Proof. Here, we prove (i), (iii), (v), (vii), (ix) and (xiii). Others can be proved analogously.

(i) Since

$$
\begin{aligned}
& \left(\left(A \boxplus_{F} B\right) \rightarrow(A @ B)^{C}\right)^{C}=\left[\min \left\{\sqrt[3]{\zeta_{a_{i j}}^{3}+\zeta_{a_{i j}}^{3}-\zeta_{a_{i j}}^{3} \zeta_{a_{i j}}^{3}}, \sqrt[3]{\frac{\zeta_{a_{i j}}^{3}+\zeta_{b_{i j}}^{3}}{2}}\right\}, \max \left\{\delta_{a_{i j}} \delta_{b_{i j}}, \sqrt[3]{\frac{\delta_{a_{i j}}^{3}+\delta_{b_{i j}}^{3}}{2}}\right\}\right] \\
& =\left[\left\langle\sqrt[3]{\frac{\zeta_{a_{i j}}^{3}+\zeta_{b_{i j}}^{3}}{2}}, \sqrt[3]{\frac{\delta_{a_{i j}}^{3}+\delta_{b_{i j}}^{3}}{2}}\right\rangle\right] \\
& =A @ B \text {, }
\end{aligned}
$$


and

$$
\begin{aligned}
\left((A @ B) \rightarrow\left(A \boxplus_{F} B\right)^{C}\right)^{C} & =\left[\min \left\{\sqrt[3]{\frac{\zeta_{a_{i j}}^{3}+\zeta_{b_{i j}}^{3}}{2}}, \sqrt[3]{\zeta_{a_{i j}}^{3}+\zeta_{a_{i j}}^{3}-\zeta_{a_{i j}}^{3} \zeta_{a_{i j}}^{3}}\right\}, \max \left\{\sqrt[3]{\frac{\delta_{a_{i j}}^{3}+\delta_{b_{i j}}^{3}}{2}}, \delta_{a_{i j}} \delta_{b_{i j}}\right\}\right] \\
& =\left[\left\langle\sqrt[3]{\frac{\zeta_{a_{i j}}^{3}+\zeta_{b_{i j}}^{3}}{2}}, \sqrt[3]{\left.\frac{\delta_{a_{i j}}^{3}+\delta_{b_{i j}}^{3}}{2}\right\rangle}\right]\right. \\
& =A @ B .
\end{aligned}
$$

So,

$$
\left(\left(A \boxplus_{F} B\right) \rightarrow(A @ B)^{C}\right)^{C}=\left((A @ B) \rightarrow\left(A \boxplus_{F} B\right)^{C}\right)^{C}=(A @ B) .
$$

(iii) Since

$$
\begin{aligned}
& \left(\left(A \otimes_{F} B\right) \rightarrow(A @ B)^{C}\right)^{C}=\left[\min \left\{\zeta_{a_{i j}} \zeta_{b_{i j}}, \sqrt[3]{\frac{\zeta_{a_{i j}}^{3}+\zeta_{b_{i j}}^{3}}{2}}\right\}, \max \left\{\sqrt[3]{\delta_{a_{i j}}^{3}+\delta_{b_{i j}}^{3}-\delta_{a_{i j}}^{3} \delta_{b_{i j}}^{3}}, \sqrt[3]{\frac{\delta_{a_{i j}}^{3}+\delta_{b_{i j}}^{3}}{2}}\right\}\right] \\
& =\left[\left\langle\zeta_{a_{i j}} \zeta_{b_{i j}}, \sqrt[3]{\delta_{a_{i j}}^{3}+\delta_{b_{i j}}^{3}-\delta_{a_{i j}}^{3} \delta_{b_{i j}}^{3}}\right\rangle\right] \\
& =A \nabla_{F} B \text {, }
\end{aligned}
$$

and

$$
\begin{aligned}
\left((A @ B) \rightarrow\left(A \bigotimes_{F} B\right)^{C}\right)^{C} & =\left[\min \left\{\sqrt[3]{\frac{\zeta_{a_{i j}}^{3}+\zeta_{b_{i j}}^{3}}{2}}, \zeta_{a_{i j}} \zeta_{b_{i j}}\right\}, \max \left\{\sqrt[3]{\frac{\delta_{a_{i j}}^{3}+\delta_{b_{i j}}^{3}}{2}}, \sqrt[3]{\delta_{a_{i j}}^{3}+\delta_{b_{i j}}^{3}-\delta_{a_{i j}}^{3} \delta_{b_{i j}}^{3}}\right\}\right] \\
& =\left[\left\langle\zeta_{a_{i j}} \zeta_{b_{i j}}, \sqrt[3]{\delta_{a_{i j}}^{3}+\delta_{a_{i j}}^{3}-\delta_{a_{i j}}^{3} \delta_{a_{i j}}^{3}}\right\rangle\right] \\
& =A \bigotimes_{F} B .
\end{aligned}
$$

So,

$$
\left(\left(A \bigotimes_{F} B\right) \rightarrow(A @ B)^{C}\right)^{C}=\left((A @ B) \rightarrow\left(A \bigotimes_{F} B\right)^{C}\right)^{C}=\left(A \bigotimes_{F} B\right)
$$

(v) Since

$$
\begin{aligned}
\left(\left(A \boxplus_{F} B\right) \rightarrow(A \# B)^{C}\right)^{C} & =\left[\min \left\{\sqrt[3]{\zeta_{a_{i j}}^{3}+\zeta_{b_{i j}}^{3}-\zeta_{a_{i j}}^{3} \zeta_{b_{i j}}^{3}}, \frac{\sqrt[3]{2} \zeta_{a_{i j}} \zeta_{b_{i j}}}{\sqrt[3]{\zeta_{a_{i j}}^{3}+\zeta_{b_{i j}}^{3}}}\right\}, \max \left\{\delta_{a_{i j}} \delta_{b_{i j}} \frac{\sqrt[3]{2} \delta_{a_{i j}} \delta_{b_{i j}}}{\sqrt[3]{\delta_{a_{i j}}^{3}+\delta_{b_{i j}}^{3}}}\right\}\right] \\
& =\left[\left\langle\frac{\sqrt[3]{2} \zeta_{a_{i j}} \zeta_{b_{i j}}}{\sqrt[3]{\zeta_{a_{i j}}^{3}+\zeta_{b_{i j}}^{3}}}, \frac{\sqrt[3]{2} \delta_{a_{i j}} \delta_{b_{i j}}}{\sqrt[3]{\delta_{a_{i j}}^{3}+\delta_{b_{i j}}^{3}}}\right\rangle\right] \\
& =A \# B
\end{aligned}
$$

and

$$
\begin{aligned}
\left((A \# B) \rightarrow\left(A \boxplus_{F} B\right)^{C}\right)^{C} & =\left[\min \left\{\frac{\sqrt[3]{2} \zeta_{a_{i j}} \zeta_{b_{i j}}}{\sqrt[3]{\zeta_{a_{i j}}^{3}+\zeta_{b_{i j}}^{3}}}, \sqrt[3]{\zeta_{a_{i j}}^{3}+\zeta_{b_{i j}}^{3}-\zeta_{a_{i j}}^{3} \zeta_{b_{i j}}^{3}}\right\}, \max \left\{\frac{\sqrt[3]{2} \delta_{a_{i j}} \delta_{b_{i j}}}{\sqrt[3]{\delta_{a_{i j}}^{3}+\delta_{b_{i j}}^{3}}}, \delta_{a_{i j}} \delta_{b_{i j}}\right\}\right] \\
& =\left[\left\langle\frac{\sqrt[3]{2} \zeta_{a_{i j}} \zeta_{b_{i j}}}{\sqrt[3]{\zeta_{a_{i j}}^{3}+\zeta_{b_{i j}}^{3}}}, \frac{\sqrt[3]{2} \delta_{a_{i j}} \delta_{b_{i j}}}{\sqrt[3]{\delta_{a_{i j}}^{3}+\delta_{b_{i j}}^{3}}}\right\rangle\right] \\
& =A \# B .
\end{aligned}
$$

So,

$$
\left(\left(A \boxplus_{F} B\right) \rightarrow(A \# B)^{C}\right)^{C}=\left((A \# B) \rightarrow\left(A \boxplus_{F} B\right)^{C}\right)^{C}=(A \# B) .
$$


(vii) Since

$$
\begin{aligned}
\left(\left(A \bigotimes_{F} B\right) \rightarrow(A \# B)^{C}\right)^{C} & =\left[x, \min \left\{\zeta_{a_{i j}} \zeta_{b_{i j}}, \frac{\sqrt[3]{2} \zeta_{a_{i j}} \zeta_{b_{i j}}}{\sqrt[3]{\delta_{a_{i j}}^{3}+\delta_{b_{i j}}^{3}}}\right\}, \max \left\{\sqrt[3]{\delta_{a_{i j}}^{3}+\delta_{b_{i j}}^{3}-\delta_{a_{i j}}^{3} \delta_{b_{i j}}^{3}}, \frac{\sqrt[3]{2} \delta_{a_{i j}} \delta_{b_{i j}}}{\sqrt[3]{\delta_{a_{i j}}^{3}+\delta_{b_{i j}}^{3}}}\right\}\right] \\
& =\left[\left\langle\zeta_{a_{i j}} \zeta_{b_{i j}}, \sqrt[3]{\delta_{a_{i j}}^{3}+\delta_{b_{i j}}^{3}-\delta_{a_{i j}}^{3} \delta_{b_{i j}}^{3}}\right\rangle\right] \\
& =A \bigotimes_{F} B
\end{aligned}
$$

and

$$
\begin{aligned}
\left((A \# B) \rightarrow\left(A \otimes_{F} B\right)^{C}\right)^{C} & =\left[x, \min \left\{\frac{\sqrt[3]{2} \zeta_{a_{i j}} \zeta_{b_{i j}}}{\sqrt[3]{\delta_{a_{i j}}^{3}+\delta_{b_{i j}}^{3}}}, \zeta_{a_{i j}} \zeta_{b_{i j}}\right\}, \max \left\{\frac{\sqrt[3]{2} \delta_{a_{i j}} \delta_{b_{i j}}}{\sqrt[3]{\delta_{a_{i j}}^{3}+\delta_{b_{i j}}^{3}}}, \sqrt[3]{\delta_{a_{i j}}^{3}+\delta_{b_{i j}}^{3}-\delta_{a_{i j}}^{3} \delta_{b_{i j}}^{3}}\right\}\right] \\
& =\left[\left\langle\zeta_{a_{i j}} \zeta_{b_{i j}}, \sqrt[3]{\delta_{a_{i j}}^{3}+\delta_{b_{i j}}^{3}-\delta_{a_{i j}}^{3} \delta_{b_{i j}}^{3}}\right\rangle\right] \\
& =A \bigotimes_{F} B .
\end{aligned}
$$

So,

$$
\left(\left(A \nabla_{F} B\right) \rightarrow(A \# B)^{C}\right)^{C}=\left((A \# B) \rightarrow\left(A \nabla_{F} B\right)^{C}\right)^{C}=\left(A \nabla_{F} B\right)
$$

(ix) Since

$$
\begin{aligned}
\left(\left(A \boxplus_{F} B\right) \rightarrow(A \$ B)^{C}\right)^{C} & =\left[\min \left\{\sqrt[3]{\zeta_{a_{i j}}^{3}+\zeta_{b_{i j}}^{3}-\zeta_{a_{i j}}^{3} \zeta_{b_{i j}}^{3}} \sqrt[3]{\zeta_{a_{i j}} \zeta_{b_{i j}}}\right\}, \max \left\{\delta_{a_{i j}} \delta_{b_{i j}}, \sqrt[3]{\delta_{a_{i j}} \delta_{b_{i j}}}\right\}\right] \\
& =\left[\left\langle\sqrt[3]{\zeta_{a_{i j}} \zeta_{b_{i j}}} \sqrt[3]{\delta_{a_{i j}} \delta_{b_{i j}}}\right\rangle\right] \\
& =A \$ B
\end{aligned}
$$

and

$$
\begin{aligned}
& \left((A \$ B) \rightarrow\left(A \boxplus_{F} B\right)^{C}\right)^{C}=\left[x, \min \left\{\sqrt[3]{\zeta_{a_{i j}} \zeta_{a_{i j}}} \sqrt[3]{\zeta_{a_{i j}}^{3}+\zeta_{a_{i j}}^{3}-\zeta_{a_{i j}}^{3} j_{a_{i j}}^{3}}\right\}, \max \left\{\sqrt[3]{\delta_{a_{i j}} \delta_{b_{i j}}} \delta_{a_{i j}} \delta_{b_{i j}}\right\}\right] \\
& =\left[\left\langle\sqrt[3]{\zeta_{a_{i j}} \zeta_{b_{i j}}}, \sqrt[3]{\delta_{a_{i j}} \delta_{b_{i j}}}\right\rangle\right] \\
& =A \$ B \text {. }
\end{aligned}
$$

So,

$$
\left(\left(A \boxplus_{F} B\right) \rightarrow(A \$ B)^{C}\right)^{C}=\left((A \$ B) \rightarrow\left(A \boxplus_{F} B\right)^{C}\right)^{C}=(A \$ B) .
$$

(xi) Since

$$
\begin{aligned}
\left(\left(A \bigotimes_{F} B\right) \rightarrow(A \$ B)^{C}\right)^{C} & =\left[\min \left\{\zeta_{a_{i j}} \zeta_{b_{i j}}, \sqrt[3]{\zeta_{a_{i j}} \zeta_{b_{i j}}}\right\}, \max \left\{\sqrt[3]{\delta_{a_{i j}}^{3}+\delta_{a_{i j}}^{3}-\delta_{a_{i j}}^{3} \delta_{a_{i j}}^{3}} \sqrt[3]{\delta_{a_{i j}} \delta_{b_{i j}}}\right\}\right] \\
& =\left[\left\langle\zeta_{a_{i j}} \zeta_{b_{i j}}, \sqrt[3]{\delta_{a_{i j}}^{3}+\delta_{a_{i j}}^{3}-\delta_{a_{i j}}^{3} \delta_{a_{i j}}^{3}}\right\rangle\right] \\
& =A \bigotimes_{F} B
\end{aligned}
$$

and

$$
\begin{aligned}
\left((A \$ B) \rightarrow\left(A \otimes_{F} B\right)^{C}\right)^{C} & =\left[\min \left\{\sqrt[3]{\zeta_{a_{i j}} \zeta_{b_{i j}}} \zeta_{a_{i j}} \zeta_{b_{i j}}\right\}, \max \left\{\sqrt[3]{\delta_{a_{i j}} \delta_{b_{i j}}}, \sqrt[3]{\delta_{a_{i j}}^{3}+\delta_{b_{i j}}^{3}-\delta_{a_{i j}}^{3} \delta_{b_{i j}}^{3}}\right\}\right] \\
& =\left[\left\langle\zeta_{a_{i j}} \zeta_{b_{i j}}, \sqrt[3]{\delta_{a_{i j}}^{3}+\delta_{b_{i j}}^{3}-\delta_{a_{i j}}^{3} \delta_{b_{i j}}^{3}}\right\rangle\right] \\
& =A \nabla_{F} B .
\end{aligned}
$$

So,

$$
\left(\left(A \nabla_{F} B\right) \rightarrow(A \$ B)^{C}\right)^{C}=\left((A \$ B) \rightarrow\left(A \nabla_{F} B\right)^{C}\right)^{C}=\left(A \bigotimes_{F} B\right)
$$


(xiii) Since

$$
\begin{aligned}
\left(\left(A \boxplus_{F} B\right) \rightarrow\left(A \otimes_{F} B\right)^{C}\right)^{C} & =\left[\min \left\{\sqrt[3]{\zeta_{a_{i j}}^{3}+\zeta_{b_{i j}}^{3}-\zeta_{a_{i j}}^{3} \zeta_{b_{i j}}^{3}} \zeta_{a_{i j}} \zeta_{b_{i j}}\right\}, \max \left\{\delta_{a_{i j}} \delta_{b_{i j}}, \sqrt[3]{\delta_{a_{i j}}^{3}+\delta_{b_{i j}}^{3}-\delta_{a_{i j}}^{3} \delta_{b_{i j}}^{3}}\right\}\right] \\
& =\left[\left\langle\zeta_{a_{i j}} \zeta_{b_{i j}}, \sqrt[3]{\delta_{a_{i j}}^{3}+\delta_{b_{i j}}^{3}-\delta_{a_{i j}}^{3} \delta_{b_{i j}}^{3}}\right\rangle\right] \\
& =A \otimes_{F} B,
\end{aligned}
$$

and

$$
\begin{aligned}
\left(\left(A \otimes_{F} B\right) \rightarrow\left(A \boxplus_{F} B\right)^{C}\right)^{C} & =\left[\min \left\{\zeta_{a_{i j}} \zeta_{b_{i j}} \sqrt[3]{\zeta_{a_{i j}}^{3}+\zeta_{b_{i j}}^{3}-\zeta_{a_{i j}}^{3} \zeta_{b_{i j}}^{3}}\right\}, \max \left\{\sqrt[3]{\delta_{a_{i j}}^{3}+\delta_{b_{i j}}^{3}-\delta_{a_{i j}}^{3} \delta_{b_{i j}}^{3}} \delta_{a_{i j}} \delta_{b_{i j}}\right\}\right] \\
& =\left[\left\langle\zeta_{a_{i j}} \zeta_{b_{i j}}, \sqrt[3]{\delta_{a_{i j}}^{3}+\delta_{b_{i j}}^{3}-\delta_{a_{i j}}^{3} \delta_{b_{i j}}^{3}}\right\rangle\right] \\
& =A \boxplus_{F} B .
\end{aligned}
$$

So,

$$
\left(\left(A \boxplus_{F} B\right) \rightarrow\left(A \otimes_{F} B\right)^{C}\right)^{C}=\left(\left(A \nabla_{F} B\right) \rightarrow\left(A \boxplus_{F} B\right)^{C}\right)^{C}=\left(A \nabla_{F} B\right)
$$

The proof of the following Corollaries follows from Theorem 9.

Corollary 10. For $A, B \in F_{m \times n}$, we have $\left(\left(A \nabla_{F} B\right) \rightarrow(A @ B)^{C}\right)^{C}=\left((A @ B) \rightarrow\left(A \bigotimes_{F} B\right)^{C}\right)^{C}=$ $\left(\left(A \bigotimes_{F} B\right) \rightarrow(A \# B)^{C}\right)^{C}=\left((A \# B) \rightarrow\left(A \bigotimes_{F} B\right)^{C}\right)^{C}=\left(\left(A \bigotimes_{F} B\right) \rightarrow(A \$ B)^{C}\right)^{C}=\left((A \$ B) \rightarrow\left(A \bigotimes_{F} B\right)^{C}\right)^{C}=$ $\left(\left(A \boxplus_{F} B\right) \rightarrow\left(A \nabla_{F} B\right)^{C}\right)^{C}=\left(\left(A \nabla_{F} B\right) \rightarrow\left(A \boxplus_{F} B\right)^{C}\right)^{C}=\left(A \nabla_{F} B\right)$.

Corollary 11. For $A, B \in F_{m \times n}$, we have $\left(\left(A \boxplus_{F} B\right)^{C} \rightarrow(A @ B)\right)=\left((A @ B)^{C} \rightarrow\left(A \boxplus_{F} B\right)\right)=$ $\left(\left(A \boxplus_{F} B\right)^{C} \rightarrow(A \# B)\right)=\left((A \# B)^{C} \rightarrow\left(A \boxplus_{F} B\right)\right)=\left(\left(A \boxplus_{F} B\right)^{C} \rightarrow(A \$ B)\right)=\left((A \$ B)^{C} \rightarrow\left(A \boxplus_{F} B\right)\right)=$ $\left(\left(A \nabla_{F} B\right)^{C} \rightarrow\left(A \boxplus_{F} B\right)\right)=\left(\left(A \boxplus_{F} B\right)^{C} \rightarrow\left(A \nabla_{F} B\right)\right)=\left(A \boxplus_{F} B\right)$.

Theorem 12. For $A, B \in F_{m \times n}$, we have $\left[\left(A^{C} \rightarrow B\right) \boxplus_{F}\left(A \rightarrow B^{C}\right)^{C}\right] @\left[\left(A^{C} \rightarrow B\right) \bigotimes_{F}\left(A \rightarrow B^{C}\right)^{C}\right]=(A @ B)$.

Proof. Since

$$
\left[\left(A^{C} \rightarrow B\right) \boxplus_{F}\left(A \rightarrow B^{C}\right)^{C}\right]=\left[\left\langle\sqrt[3]{\zeta_{a_{i j}}^{3}+\zeta_{b_{i j}}^{3}-\zeta_{a_{i j}}^{3} \zeta_{b_{i j}}^{3}} \delta_{a_{i j}} \delta_{b_{i j}}\right\rangle\right]
$$

and

$$
\left[\left(A^{C} \rightarrow B\right) \otimes_{F}\left(A \rightarrow B^{C}\right)^{C}\right]=\left[\left\langle\zeta_{a_{i j}} \zeta_{b_{i j}}, \sqrt[3]{\delta_{a_{i j}}^{3}+\delta_{b_{i j}}^{3}-\delta_{a_{i j}}^{3} \delta_{b_{i j}}^{3}}\right\rangle\right]
$$

So,

$$
\begin{aligned}
& {\left[\left(A^{C} \rightarrow B\right) \boxplus_{F}\left(A \rightarrow B^{C}\right)^{C}\right] @\left[\left(A^{C} \rightarrow B\right) \otimes_{F}\left(A \rightarrow B^{C}\right)^{C}\right]} \\
& =\left[\sqrt[3]{\frac{\left(\sqrt[3]{\zeta_{a_{i j}}^{3}+\zeta_{b_{i j}}^{3}-\zeta_{a_{i j}}^{3} \zeta_{b_{i j}}^{3}}\right)^{3}+\zeta_{a_{i j}}^{3} \zeta_{b_{i j}}^{3}}{2}}, \sqrt[3]{\frac{\delta_{a_{i j}}^{3} \delta_{b_{i j}}^{3}+\left(\sqrt[3]{\delta_{a_{i j}}^{3}+\delta_{b_{i j}}^{3}-\delta_{a_{i j}}^{3} \delta_{b_{i j}}^{3}}\right)^{3}}{2}}\right] \\
& =\left[\left\langle\sqrt[3]{\frac{\zeta_{a_{i j}}^{3}+\zeta_{b_{i j}}^{3}}{2}}, \sqrt[3]{\frac{\delta_{a_{i j}}^{3}+\delta_{b_{i j}}^{3}}{2}}\right\rangle\right] \\
& =(A @ B) \text {. }
\end{aligned}
$$


Theorem 13. For $A, B \in F_{m \times n}$, we have

$$
\begin{aligned}
{\left[\left(\left(A^{C} \rightarrow B\right)\right.\right.} & \left.\left.\boxplus_{F}\left(A \rightarrow B^{C}\right)^{C}\right) \cap\left(\left(A^{C} \rightarrow B\right) \otimes_{F}\left(A \rightarrow B^{C}\right)^{C}\right)\right] \\
& @\left[\left(\left(A^{C} \rightarrow B\right) \boxplus_{F}\left(A \rightarrow B^{C}\right)^{C}\right) \cup\left(\left(A^{C} \rightarrow B\right) \otimes_{F}\left(A \rightarrow B^{C}\right)^{C}\right)\right]=(A @ B) .
\end{aligned}
$$

Proof. Since

$$
\begin{aligned}
{\left[\left(\left(A^{C} \rightarrow B\right)\right.\right.} & \left.\left.\boxplus_{F}\left(A \rightarrow B^{C}\right)^{C}\right) \cap\left(\left(A^{C} \rightarrow B\right) \bigotimes_{F}\left(A \rightarrow B^{C}\right)^{C}\right)\right] \\
& =\left[\min \left\{\sqrt[3]{\zeta_{a_{i j}}^{3}+\zeta_{b_{i j}}^{3}-\zeta_{a_{i j}}^{3} \zeta_{b_{i j}}^{3}} \zeta_{a_{i j}} \zeta_{b_{i j}}\right\}, \max \left\{\delta_{a_{i j}} \delta_{a_{i j}}, \sqrt[3]{\delta_{a_{i j}}^{3}+\delta_{b_{i j}}^{3}-\delta_{a_{i j}}^{3} \delta_{b_{i j}}^{3}}\right\}\right] \\
& =\left[\left\langle\zeta_{a_{i j}} \zeta_{b_{i j}}, \sqrt[3]{\delta_{a_{i j}}^{3}+\delta_{b_{i j}}^{3}-\delta_{a_{i j}}^{3} \delta_{b_{i j}}^{3}}\right\rangle\right]
\end{aligned}
$$

and

$$
\begin{aligned}
{\left[\left(\left(A^{C} \rightarrow B\right)\right.\right.} & \left.\left.\boxplus_{F}\left(A \rightarrow B^{C}\right)^{C}\right) \cup\left(\left(A^{C} \rightarrow B\right) \otimes_{F}\left(A \rightarrow B^{C}\right)^{C}\right)\right] \\
& =\left[\max \left\{\sqrt[3]{\zeta_{a_{i j}}^{3}+\zeta_{b_{i j}}^{3}-\zeta_{a_{i j}}^{3} \zeta_{b_{i j}}^{3}} \zeta_{a_{i j}} \zeta_{b_{i j}}\right\}, \min \left\{\delta_{a_{i j}} \delta_{b_{i j}} \sqrt[3]{\delta_{a_{i j}}^{3}+\delta_{b_{i j}}^{3}-\delta_{a_{i j}}^{3} \delta_{b_{i j}}^{3}}\right\}\right] \\
& =\left[\left\langle\sqrt[3]{\zeta_{a_{i j}}^{3}+\zeta_{b_{i j}}^{3}-\zeta_{a_{i j}}^{3} \zeta_{b_{i j}}^{3}} \delta_{a_{i j}} \delta_{b_{i j}}\right\rangle\right] .
\end{aligned}
$$

Hence

$$
\begin{aligned}
& {\left[\left(\left(A^{C} \rightarrow B\right) \boxplus_{F}\left(A \rightarrow B^{C}\right)^{C}\right) \cap\left(\left(A^{C} \rightarrow B\right) \otimes_{F}\left(A \rightarrow B^{C}\right)^{C}\right)\right]} \\
& @\left[\left(\left(A^{C} \rightarrow B\right) \boxplus_{F}\left(A \rightarrow B^{C}\right)^{C}\right) \cup\left(\left(A^{C} \rightarrow B\right) \rrbracket_{F}\left(A \rightarrow B^{C}\right)^{C}\right)\right]
\end{aligned}
$$

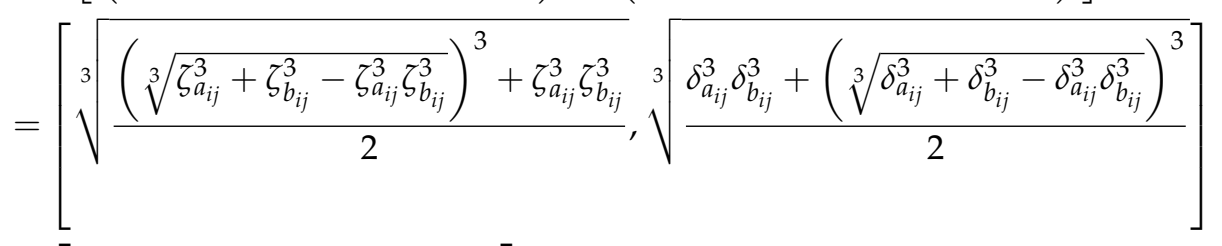

$$
\begin{aligned}
& =\left[\left\langle\sqrt[3]{\frac{\zeta_{a_{i j}}^{3}+\zeta_{b_{i j}}^{3}}{2}}, \sqrt[3]{\frac{\delta_{a_{i j}+\delta_{b_{i j}}^{3}}^{3}}{2}}\right\rangle\right] \\
& =A @ B \text {. }
\end{aligned}
$$

Theorem 14. For $A, B \in F_{m \times n}$, we have

$\left[\left(\left(A \boxplus_{F} B\right) \rightarrow(A @ B)^{C}\right)^{C} \cup\left(\left(A \rrbracket_{F} B\right) \rightarrow(A @ B)^{C}\right)^{C}\right] \cup\left[\left(\left(A \boxplus_{F} B\right) \rightarrow(A @ B)^{C}\right)^{C} \cap\left(\left(A \bigotimes_{F} B\right) \rightarrow(A @ B)^{C}\right)^{C}\right]=$ $A @ B$.

Proof. From Theorem 9, we have

$$
\left(\left(A \boxplus_{F} B\right) \rightarrow(A @ B)^{C}\right)^{C}=\left[\left\langle\sqrt[3]{\frac{\zeta_{a_{i j}}^{3}+\zeta_{b_{i j}}^{3}}{2}}, \sqrt[3]{\left.\frac{\delta_{a_{i j}}^{3}+\delta_{b_{i j}}^{3}}{2}\right\rangle}\right]\right.
$$

and

$$
\left(\left(A \bigotimes_{F} B\right) \rightarrow(A @ B)^{C}\right)^{C}=\left[\left\langle\zeta_{a_{i j}} \zeta_{b_{i j}}, \sqrt[3]{\delta_{a_{i j}}^{3}+\delta_{a_{i j}}^{3}-\delta_{a_{i j}}^{3} \delta_{a_{i j}}^{3}}\right\rangle\right]
$$


So,

$$
\begin{aligned}
{\left[\left(\left(A \boxplus_{F} B\right)\right.\right.} & \left.\left.\rightarrow(A @ B)^{C}\right)^{C} \cup\left(\left(A \bigotimes_{F} B\right) \rightarrow(A @ B)^{C}\right)^{C}\right] \\
& =\left[\max \left\{\sqrt[3]{\frac{\zeta_{a_{i j}}^{3}+\zeta_{b_{i j}}^{3}}{2}},\left(\zeta_{a_{i j}} \zeta_{b_{i j}}\right)\right\}, \min \left\{\sqrt[3]{\left.\left.\frac{\delta_{a_{i j}+\delta_{b_{i j}}^{3}}^{3}}{2}, \sqrt[3]{\delta_{a_{i j}}^{3}+\delta_{b_{i j}}^{3}-\delta_{a_{i j}}^{3} \delta_{b_{i j}}^{3}}\right\}\right]}\right.\right. \\
& =\left[\left\langle\sqrt[3]{\frac{\zeta_{a_{i j}}^{3}+\zeta_{b_{i j}}^{3}}{2}}, \sqrt[3]{\left.\left.\frac{\delta_{a_{i j}}^{3}+\delta_{b_{i j}}^{3}}{2}\right\rangle\right]},\right.\right.
\end{aligned}
$$

and

$$
\begin{aligned}
{\left[\left(\left(A \boxplus_{F} B\right)\right.\right.} & \text { rightarrow } \left.\left.(A @ B)^{C}\right)^{C} \cap\left(\left(A \bigotimes_{F} B\right) \rightarrow(A @ B)^{C}\right)^{C}\right] \\
& =\left[\min \left\{\sqrt[3]{\frac{\zeta_{a_{i j}}^{3}+\zeta_{b_{i j}}^{3}}{2}},\left(\zeta_{a_{i j}} \zeta_{b_{i j}}\right)\right\}, \max \left\{\sqrt[3]{\frac{\delta_{a_{i j}}^{3}+\delta_{b_{i j}}^{3}}{2}}, \sqrt[3]{\delta_{a_{i j}}^{3}+\delta_{b_{i j}}^{3}-\delta_{a_{i j}}^{3} \delta_{b_{i j}}^{3}}\right\}\right] \\
& =\left[\left\langle\zeta_{a_{i j}} \zeta_{b_{i j}}, \sqrt[3]{\delta_{a_{i j}}^{3}+\delta_{b_{i j}}^{3}-\delta_{a_{i j}}^{3} \delta_{b_{i j}}^{3}}\right\rangle\right] .
\end{aligned}
$$

Hence

$$
\begin{aligned}
& {\left[\left(\left(A \boxplus_{F} B\right) \rightarrow(A @ B)^{C}\right)^{C} \cup\left(\left(A \otimes_{F} B\right) \rightarrow(A @ B)^{C}\right)^{C}\right]\left[\left(\left(A \boxplus_{F} B\right) \rightarrow(A @ B)^{C}\right)^{C} \cap\left(\left(A \otimes_{F} B\right) \rightarrow(A @ B)^{C}\right)^{C}\right]} \\
& =\left[\max \left\{\sqrt[3]{\frac{\zeta_{a_{i j}}^{3}+\zeta_{b_{i j}}^{3}}{2}},\left(\zeta_{a_{i j}} \zeta_{b_{i j}}\right)\right\}, \min \left\{\sqrt[3]{\frac{\delta_{a_{i j}}^{3}+\delta_{b_{i j}}^{3}}{2}}, \sqrt[3]{\delta_{a_{i j}}^{3}+\delta_{b_{i j}}^{3}-\delta_{a_{i j}}^{3} b_{b_{i j}}^{3}}\right\}\right] \\
& =\left[\left\langle\sqrt[3]{\frac{\zeta_{a_{i j}}^{3}+\zeta_{b_{i j}}^{3}}{2}}, \sqrt[3]{\frac{\delta_{a_{i j}}^{3}+\delta_{b_{i j}}^{3}}{2}}\right\rangle\right]=A @ B .
\end{aligned}
$$

Theorem 15. For $A, B \in F_{m \times n}$, we have $\left[\left(\left(A \boxplus_{F} B\right) \rightarrow(A @ B)^{C}\right)^{C} \cup\left(\left(A \bigotimes_{F} B\right) \rightarrow(A @ B)^{C}\right)^{C}\right] \cap\left[\left(\left(A \boxplus_{F} B\right) \rightarrow(A @ B)^{C}\right)^{C} \cap\left(\left(A \bigotimes_{F} B\right) \rightarrow(A @ B)^{C}\right)^{C}\right]=$
$A \bigotimes_{F} B$.

Proof. The proof is similar to that of Theorem 14.

Theorem 16. For $A, B \in F_{m \times n}$, we have $\left(\left(A \boxplus_{F} B\right)^{C} \rightarrow(A @ B)\right) @\left(\left(A \bigotimes_{F} B\right) \rightarrow(A @ B)^{C}\right)^{C}=(A @ B)$.

Proof. Since

$$
\left(\left(A \boxplus_{F} B\right)^{C} \rightarrow(A @ B)\right)=\left[\left\langle\sqrt[3]{\zeta_{a_{i j}}^{3}+\zeta_{b_{i j}}^{3}-\zeta_{a_{i j}}^{3} \zeta_{b_{i j}}^{3}} \delta_{a_{i j}} \delta_{b_{i j}}\right\rangle\right]
$$

and

$$
\left(\left(A \otimes_{F} B\right) \rightarrow(A @ B)^{C}\right)^{C}=\left[\left\langle\zeta_{a_{i j}} \zeta_{b_{i j}}, \sqrt[3]{\delta_{a_{i j}}^{3}+\delta_{b_{i j}}^{3}-\delta_{a_{i j}}^{3} \delta_{b_{i j}}^{3}}\right\rangle\right]
$$

So,

$$
\begin{aligned}
\left(\left(A \boxplus_{F} B\right)^{C}\right. & \rightarrow(A @ B)) @\left(\left(A \nabla_{F} B\right) \rightarrow(A @ B)^{C}\right)^{C} \\
& =\left[\sqrt[3]{\frac{\left(\sqrt[3]{\zeta_{a_{i j}}^{3}+\zeta_{b_{i j}}^{3}-\zeta_{a_{i j}}^{3} \zeta_{b_{i j}}^{3}}\right)^{3}+\left(\zeta_{a_{i j}} \zeta_{b_{i j}}\right)^{3}}{2}}, \sqrt[3]{\frac{\left(\delta_{a_{i j}} \delta_{b_{i j}}\right)^{3}+\left(\sqrt[3]{\delta_{a_{i j}}^{3}+\delta_{b_{i j}}^{3}-\delta_{a_{i j}}^{3} \delta_{b_{i j}}^{3}}\right)^{3}}{2}}\right]
\end{aligned}
$$




$$
=\left[\left\langle\sqrt[3]{\frac{\zeta_{a_{i j}}^{3}+\zeta_{b_{i j}}^{3}}{2}}, \sqrt[3]{\frac{\delta_{a_{i j}}^{3}+\delta_{b_{i j}}^{3}}{2}}\right\rangle\right]=A @ B
$$

Theorem 17. For $A, B \in F_{m \times n}$, we have $\left(\left(A \boxplus_{F} B\right)^{C} \rightarrow(A \# B)\right) @\left(\left(A \bigotimes_{F} B\right) \rightarrow(A \# B)^{C}\right)^{C}=(A @ B)$.

Proof. Since

$$
\left(\left(A \boxplus_{F} B\right)^{C} \rightarrow(A \# B)\right)=\left[\left\langle\sqrt[3]{\zeta_{a_{i j}}^{3}+\zeta_{b_{i j}}^{3}-\zeta_{a_{i j}}^{3} \zeta_{b_{i j}}^{3}} \delta_{a_{i j}} \delta_{b_{i j}}\right\rangle\right]
$$

and

$$
\left(\left(A \otimes_{F} B\right) \rightarrow(A \# B)^{C}\right)^{C}=\left[\left\langle\zeta_{a_{i j}} \zeta_{b_{i j}}, \sqrt[3]{\delta_{a_{i j}}^{3}+\delta_{b_{i j}}^{3}-\delta_{a_{i j}}^{3} \delta_{b_{i j}}^{3}}\right\rangle\right] .
$$

So,

$$
\begin{aligned}
\left(\left(A \boxplus_{F} B\right)^{C}\right. & \rightarrow(A \# B)) @\left(\left(A \boxplus_{F} B\right) \rightarrow(A \# B)^{C}\right)^{C} \\
& =\left[\sqrt[3]{\frac{\left(\sqrt[3]{\zeta_{a_{i j}}^{3}+\zeta_{b_{i j}}^{3}-\zeta_{a_{i j}}^{3} \zeta_{b_{i j}}^{3}}\right)^{3}+\left(\zeta_{a_{i j}} \zeta_{b_{i j}}\right)^{3}}{2}}, \sqrt[3]{\frac{\left(\delta_{a_{i j}} \delta_{b_{i j}}\right)^{3}+\left(\sqrt[3]{\delta_{a_{i j}}^{3}+\delta_{b_{i j}}^{3}-\delta_{a_{i j}}^{3} \delta_{b_{i j}}^{3}}\right)^{3}}{2}}\right] \\
& =\left[\left\langle\sqrt[3]{\frac{\zeta_{a_{i j}}^{3}+\zeta_{b_{i j}}^{3}}{2}}, \sqrt[3]{\frac{\delta_{a_{i j}}^{3}+\delta_{b_{i j}}^{3}}{2}}\right\rangle\right] \\
& =A @ B .
\end{aligned}
$$

Theorem 18. For $A, B \in F_{m \times n}$, we have $\left(\left(A \boxplus_{F} B\right)^{C} \rightarrow(A \$ B)\right) @\left(\left(A \bigotimes_{F} B\right) \rightarrow(A \$ B)^{C}\right)^{C}=(A @ B)$.

Proof. Since

$$
\left(\left(A \boxplus_{F} B\right)^{C} \rightarrow(A \$ B)\right)=\left[\left\langle\sqrt[3]{\zeta_{a_{i j}}^{3}+\zeta_{b_{i j}}^{3}-\zeta_{a_{i j}}^{3} \zeta_{b_{i j}}^{3}} \delta_{a_{i j}} \delta_{b_{i j}}\right\rangle\right]
$$

and

$$
\left(\left(A \bigotimes_{F} B\right) \rightarrow(A \$ B)^{C}\right)^{C}=\left[\left\langle\zeta_{a_{i j}} \zeta_{b_{i j}}, \sqrt[3]{\delta_{a_{i j}}^{3}+\delta_{b_{i j}}^{3}-\delta_{a_{i j}}^{3} \delta_{b_{i j}}^{3}}\right\rangle\right]
$$

So,

$$
\begin{aligned}
\left(\left(A \boxplus_{F} B\right)^{C}\right. & \rightarrow(A \$ B))\left(\left(A \bigotimes_{F} B\right) \rightarrow(A \$ B)^{C}\right)^{C} \\
& =\left[\sqrt[3]{\frac{\left(\sqrt[3]{\zeta_{a_{i j}}^{3}+\zeta_{b_{i j}}^{3}-\zeta_{a_{i j}}^{3} \zeta_{b_{i j}}^{3}}\right)^{3}+\left(\zeta_{a_{i j}} \zeta_{b_{i j}}\right)^{3}}{2}}, \sqrt[3]{\frac{\left(\delta_{a_{i j}} \delta_{b_{i j}}\right)^{3}+\left(\sqrt[3]{\delta_{a_{i j}}^{3}+\delta_{b_{i j}}^{3}-\delta_{a_{i j}}^{3} b_{b_{i j}}^{3}}\right)^{3}}{2}}\right] \\
& =\left[\left\langle\sqrt[3]{\frac{\zeta_{a_{i j}}^{3}+\zeta_{b_{i j}}^{3}}{2}}, \sqrt[3]{\left.\left.\frac{\delta_{a_{i j}}^{3}+\delta_{b_{i j}}^{3}}{2}\right\rangle\right]}\right.\right. \\
& =A @ B .
\end{aligned}
$$

Theorem 19. For $A, B \in F_{m \times n}$, we have $\left(\left(A \nabla_{F} B\right)^{C} \rightarrow\left(A \boxplus_{F} B\right)\right) @\left(\left(A \boxplus_{F} B\right) \rightarrow\left(A \bigotimes_{F} B\right)^{C}\right)^{C}=(A @ B)$.

Proof. Since

$$
\left(\left(A \boxplus_{F} B\right)^{C} \rightarrow\left(A \boxplus_{F} B\right)\right)=\left[\left\langle\sqrt[3]{\zeta_{a_{i j}}^{3}+\zeta_{b_{i j}}^{3}-\zeta_{a_{i j}}^{3} \zeta_{b_{i j}}^{3}} \delta_{a_{i j}} \delta_{b_{i j}}\right\rangle\right]
$$


and

$$
\left(\left(A \boxplus_{F} B\right) \rightarrow\left(A \otimes_{F} B\right)^{C}\right)^{C}=\left[\left\langle\zeta_{a_{i j}} \zeta_{b_{i j}} \sqrt[3]{\delta_{a_{i j}}^{3}+\delta_{b_{i j}}^{3}-\delta_{a_{i j}}^{3} \delta_{b_{i j}}^{3}}\right\rangle\right]
$$

So,

$$
\begin{aligned}
\left(\left(A \otimes_{F} B\right)^{C}\right. & \left.\rightarrow\left(A \boxplus_{F} B\right)\right)\left(\left(A \boxplus_{F} B\right) \rightarrow\left(A \boxplus_{F} B\right)^{C}\right)^{C} \\
& =\left[\sqrt[3]{\frac{\left(\sqrt[3]{\zeta_{a_{i j}}^{3}+\zeta_{b_{i j}}^{3}-\zeta_{a_{i j}}^{3} \zeta_{b_{i j}}^{3}}\right)^{3}+\left(\zeta_{a_{i j}} \zeta_{b_{i j}}\right)^{3}}{2}}, \sqrt[3]{\frac{\left(\delta_{a_{i j}} \delta_{b_{i j}}\right)^{3}+\left(\sqrt[3]{\delta_{a_{i j}}^{3}+\delta_{b_{i j}}^{3}-\delta_{a_{i j}}^{3} \delta_{b_{i j}}^{3}}\right)^{3}}{2}}\right] \\
& =\left[\left\langle\sqrt[3]{\frac{\zeta_{a_{i j}}^{3}+\zeta_{b_{i j}}^{3}}{2}}, \sqrt[3]{\frac{\delta_{a_{i j}}^{3}+\delta_{b_{i j}}^{3}}{2}}\right\rangle\right] \\
& =A @ B .
\end{aligned}
$$

\section{Conclusion and future scope}

In this paper, some new operators $[(A \$ B),(A \# B),(A * B),(A \rightarrow B)]$ of Fermatean fuzzy matrices are defined and investigated their several algebraic properties. Further, the necessity and possibility operators of Fermatean fuzzy matrices are proved. Finally, we have identified and proved several of these properties, particularly those involving the operator $A \rightarrow B$ defined as Fermatean fuzzy implication with other operators. In further research, we may apply these operators in the field of different areas, for example, dynamic decision and consensus, business and marketing management, design, engineering and manufacturing, information technology and networking applications, human resources management, military applications, energy management, geographic information system applications etc.

Conflicts of Interest: "The author declares no conflict of interest".

\section{References}

[1] Khan, S.K, Pal, M., \& Shyamal, A.K. (2002). Intuitionistic Fuzzy Matrices. Notes on Intuitionistic Fuzzy Sets, 8(2), 51-62.

[2] Im, Y.P. Lee, F.B., \& Park, S.W. (2001). The determinant of square intuitionistic fuzzy matrices. Far East Journal of Mathematical Science, 3(5), 789-796.

[3] Thomason, M.G. (1977). Convergence of powers of Fuzzy matrix. Journal of Mathematical Analysis and Applications, 57 (2), 476-480.

[4] Silambarasan, I., \& Sriram, S.(2018). Algebraic operations on Pythagorean fuzzy matrices. Mathematical Sciences International Research Journal, 7(2), 406-414.

[5] Pal, P. (2001). Intuitionistic fuzzy determinant. V.U.J. Physical Sciences, 7, 87-93.

[6] Khan, S.K., \& Pal, M. (2006). Some operations on Intuitionistic Fuzzy Matrices. Acta Ciencia Indica, 32, 515-524.

[7] Mondal, S., \& Pal, M. (2013). Similarity relations, invertibility and eigenvalues of IFM. Fuzzy Information and Engineering, 5(4), 431-443.

[8] Emam, E.G., \& Fndh, M.A. (2016). Some results associated with the max-min and min-max compositions of bifuzzy matrices. Journal of the Egyption Mathematical Society, 24(4), 515-521.

[9] Muthuraji,T., Sriram, S., \& Murugadas, P. (2016). Decomposition of intuitionistic fuzzy matrices. Fuzzy Information and Engineering, 8(3), 345-354.

[10] Sriram, S., \& Boobalan, J. (2016). Monoids of intuitionistic fuzzy matrices. Annals of fuzzy Mathematics and Informatics, 11(3), 505-510.

[11] Muthuraji, T., \& Sriram, S. (2017). Representation and decomposition of an intuitionistic fuzzy matrix using some $\left(\alpha, \alpha^{\prime}\right)$ cuts. Applications and Applied Mathematics , 12(1), 241-258.

[12] Silambarasan, I., \& Sriram, S. (2019). New operations for Pythagorean Fuzzy matrices. Indian Journal of Science and Technology, 12(20), 1-7.

[13] Silambarasan, I., \& Sriram, S. (2019). Implication operator on Pythagorean Fuzzy set. International Journal of Scientific E Technology Research, 8 (8), 1505-1509.

[14] Silambarasan, I., \& Sriram, S. (2020). Some operations over Pythagorean fuzzy matrices based on Hamacher operations. Applications and Applied Mathematics, 15(1), 353-371. 
[15] Silambarasan, I.(2020). New operators for Fermatean fuzzy sets. Annals of Communications in Mathematics, 3(2), 116-131.

[16] Silambarasan, I., \& Sriram, S. (2021). Some operations over intuitionistic fuzzy matrices based on Hamacher t-norm and t-conorm. TWMS Journal of Applied and Engineering Mathematics, 11(2), 541-551.

[17] Silambarasan, I. (2021). Some new operations and their properties on q-rung orthopair fuzzy matrices. Asia Mathematika, 5 (1), 119-133.

[18] Silambarasan, I. (2020). Fermatean fuzzy matrices. TWMS Journal of Applied and Engineering Mathematics. Accepted.

[19] Ali, Z., \& Mahmood, T. (2020). Maclaurin symmetric mean operators and their applications in the environment of complex q-rung orthopair fuzzy sets. Computational and Applied Mathematics, 39, 1-27.

[20] Ali Z., Mahmood T., \& Yang M-S.(2020). TOPSIS method based on complex spherical fuzzy sets with Bonferroni mean operators. Mathematics, 8 (10), 1739. https://doi.org/10.3390/math8101739

[21] Ali Z, Mahmood T., \& Yang M-S.(2020). Complex T-spherical fuzzy aggregation operators with application to multi-attribute decision making. Symmetry, 12(8), 1311. https://doi.org/10.3390/sym12081311

[22] Mahmood, T. (2020). A novel approach towards bipolar soft sets and their applications. Journal of Mathematics, 2020, Article ID 4690808, https:/ / doi.org/10.1155/2020/4690808. 